Revue internationale P.M.E.

Économie et gestion de la petite et moyenne entreprise

\title{
La relation technologie-territoire et les milieux innovateurs
}

\section{Bruno Lecoq}

Volume 8, numéro 1, 1995

URI : https://id.erudit.org/iderudit/1008276ar

DOI : https://doi.org/10.7202/1008276ar

Aller au sommaire du numéro

Éditeur(s)

Presses de l’Université du Québec

ISSN

0776-5436 (imprimé)

1918-9699 (numérique)

Découvrir la revue

Citer cette note

Lecoq, B. (1995). La relation technologie-territoire et les milieux innovateurs. Revue internationale P.M.E., 8(1), 81-106. https://doi.org/10.7202/1008276ar

\section{Résumé de l'article}

Cet article tente d'esquisser un bilan des principaux apports théoriques et empiriques des recherches menées depuis 1984 à partir du concept de milieu innovateur. Ce concept a été utilisé pour étudier les interactions entre les comportements innovateurs des firmes, les processus d'organisation et de dynamique industrielle et l'environnement territorial. Il sera l'occasion de réexaminer l'évolution de la problématique, de spécifier les apports et de souligner l'originalité de la démarche. Cette mise en perspective permettra de confronter les travaux sur les milieux innovateurs avec d'autres démarches, d'en souligner la complémentarité, les points d'accord et éventuellement les divergences. Cette revue des débats permettra de définir la notion de milieu innovateur, de la replacer dans une problématique plus large relative à l'analyse des dynamiques industrielles localisées pour finalement dégager de nouvelles pistes de recherches.
Ce document est protégé par la loi sur le droit d'auteur. L'utilisation des services d’Érudit (y compris la reproduction) est assujettie à sa politique d'utilisation que vous pouvez consulter en ligne.

https://apropos.erudit.org/fr/usagers/politique-dutilisation/ 


\title{
Notes de recherche
}

\section{La relation technologie-territoire et les milieux innovateurs ${ }^{1}$}

\author{
Bruno LECOQ* \\ LATAPSES-CNRS
}

MOTS CLÉS

\section{Technologie - Territoire - Firme innovatrice - Coopération \\ Organisation - Milieu innovateur - Réseau d'innovation Dynamique industrielle localisée}

\begin{abstract}
RÉSUMÉ
Cet article tente d'esquisser un bilan des principaux apports théoriques et empiriques des recherches menées depuis 1984 à partir du concept de milieu innovateur. Ce concept a été utilisé pour étudier les interactions entre les comportements innovateurs des firmes, les processus d'organisation et de dynamique industrielle et l'environnement territorial. II sera l'occasion de réexaminer l'évolution de la problématique, de spécifier les apports et de souligner l'originalité de la démarche. Cette mise en perspective permettra de
\end{abstract}

* L'auteur termine actuellement une thèse intitulée : «Dynamique industrielle et évolution des territoires : une perspective marshallienne ». Ses travaux portent essentiellement sur l'analyse économique des systèmes localisés de production et d'innovation et sur la construction territoriale de la dynamique industrielle. A publié récemment : «Dynamique industrielle, histoire et localisation: Alfred Marshall revisité », dans Revue française d'économie, vol. 8, n 4, automne 1993. Adresse : LATAPSES (Laboratoire "Transformations de l'appareil productif et stratégies économiques sectorielles »), Groupe des laboratoires du CNRS, 250, rue Albert-Einstein, Sophia-Antipolis, 06560 Valbonne, France.

1. Cette contribution prolonge une courte note de recherche publiée dans la Revue d'économie industrielle dans le cadre d'une réflexion collective autour de la notion de proximité (Bellet et al., 1992). La réflexion sur la problématique des milieux innovateurs ne prétend ni être exhaustive ni se substituer aux analyses des membres du GREMI (Groupe de recherche européen sur les milieux innovateurs). Je tiens à remercier Pierre-André Julien, Denis Maillat ainsi que trois rapporteurs anonymes pour leurs remarques et suggestions. Je reste cependant seul responsable des imperfections qui pourraient subsister. 
confronter les travaux sur les milieux innovateurs avec d'autres démarches, d'en souligner la complémentarité, les points d'accord et éventuellement les divergences. Cette revue des débats permettra de définir la notion de milieu innovateur, de la replacer dans une problématique plus large relative à l'analyse des dynamiques industrielles localisées pour finalement dégager de nouvelles pistes de recherches.

\section{ABSTRACT}

The aim of this paper is to overview the empirical and theoretical literature on the innovative milieu approach. Since 1984, the concept of innovative milieu has been used to study the interactions between the innovative behaviors of firms, the processes of industrial organization and dynamics and the territorial environment. In the first part of the paper, we examine GREMI's empirical inquiries on the link between the process of technological creation and the shaping of economic space. The second part of the paper defines the concept of innovative milieu and suggests convergences or divergences with other approaches. Finally the paper investigates the contribution of the innovative milieu approach to a theory of industrial localized dynamics and raises a number of unresolved issues.

\section{RESUMEN}

El objectivo de este articulo es presentar la literatura empírica y téorica sobre el medio innovador. Desde 1984, este concepto ha sido utilizado para estudiar las interacciones que existen entre las actitudes innovadoras de las empresas, los procesos de organización industrial y las dinámicas y el medio ambiente territorial. En la primera parte de este trabajo, examinamos las investigaciones empíricas del GREMI sobre la articulación entre el proceso de la creación tecnológica y la formación del espacio económico. La segunda parte de este trabajo define el concepto del medio innovador y sugiere las convergencias y divergencias con los otros estudios. Finalmente nuestro trabajo investiga la contribución del estudio del medio innovador por una teoría de las dinámicas industriales localizadas y extrae algunas pistas de investigaciones nuevas.

\section{Introduction}

La question des relations entre technologie et territoire a suscité ces dix dernières années une attention renouvelée. Principalement orientée sous l'angle des facteurs de localisation, l'identification de critères quantitatifs et qualitatifs tels que la proximité d'une université et de centres de recherche, la disponibilité d'une main-d'œuvre qualifiée, la présence d'un environnement naturel et résidentiel agréable ou encore l'accès rapide à une infrastructure de transport a polarisé l'essentiel de la réflexion sur la distribution spatiale des activités ou industries dites de haute technologie. Le foisonnement de ce type d'approche centré sur la détermination de facteurs de localisation et la multiplication de critères souvent hétérogènes a rapidement révélé les insuffisances et limites d'une démarche qui 
laisse en suspens un certain nombre de questions et points obscurs ${ }^{2}$. C'est précisément en réaction aux insuffisances des approches traditionnelles que l'on peut situer l'émergence des travaux du GREMI (Groupe de recherche européen sur les milieux innovateurs), ou approche par les « milieux innovateurs $»^{3}$. Selon Aydalot (1986), à l'origine de cette initiative scientifique, notre connaissance de la relation entre technologie et territoire «mêle les idées reçues, les comparaisons faciles, les hypothèses insuffisamment vérifiées et les résultats de travaux nombreux mais menés en ordre dispersé et guère comparables ». Il s'agit dès lors « de lever certaines des faiblesses conceptuelles dans la connaissance des processus de la diffusion des technologies nouvelles $»^{4}$.

2. W. Störh (1986), à ce sujet, n'hésite pas à souligner le caractère particulièrement ambigu des résultats ainsi obtenus. Il observe que " this ambiguity is mainly due to the isolated analysis and assumption of additivity of universally discrete factors while in reality innovation generally seems to be created by the mutual - and occasionally quite unique - interaction (synergy) of various of these and other factors within rather different local or regional environments ».

3. On trouvera dans Maillat et Lecoq (1992) une discussion approfondie consacrée, d'une part, aux insuffisances des schémas traditionnels de la science régionale dans l'explication des disparités spatiales et, d'autre part, à l'émergence « des nouveaux espaces industriels ». Cet article souligne, par ailleurs, l'intérêt d'une approche alternative basée sur le concept de milieu innovateur.

4. Les termes par lesquels la question des relations technologie et territoire a successivement été formulée illustrent parfaitement l'évolution de la réflexion. Initialement centrée sur la problématique standard de la diffusion spatiale des innovations, la réflexion a porté au début des années 80 sur la question de l'innovation dans le développement régional, puis sur l'étude des interdépendances entre changement technologique et territoire, pour s'ouvrir enfin au début des années 90 sur la reconnaissance et l'étude des formes territoriales de la technologie (système localisé d'innovation, district technologique...). En réalité, d'une conception de la technologie comme information et de l'espace comme distance, la réflexion théorique récente s'est portée sur une approche de la technologie et du territoire comme ressources particulières. Plus fondamentalement, ce cheminement va bien au-delà d'un aménagement du cadre d'analyse initial puisqu'il oppose une approche centrée sur une problématique d'allocation de ressources génériques à une problématique de la création de ressources particulières. Sur ces questions, on trouvera dans Bellet (1992) un excellent survey couvrant l'essentiel des débats et de la littérature sur l'analyse économique des rapports entre technologie et territoire depuis l'article pionnier de Griliches consacré à la diffusion inégale d'une innovation (le maïs hybride) au sein des régions américaines. Notons enfin que l'on retrouve cette opposition dans les travaux du GREMI. La première période correspondant à l'étape GREMI 1 (Aydalot, 1986 ; GREMI, 1987 ; Aydalot et Keeble,1988) reste fortement imprégnée par la problématique de l'allocation de ressources et la question de la diffusion spatiale des innovations. En revanche, à partir de GREMI 2 (colloque d'Arco, 1988) mais surtout GREMI 3 (colloque de Neuchâtel, 1990) la problématique de la création de ressources particulières semble nettement privilégiée sous l'influence des travaux de M. Amendola et J.L. Gaffard, travaux dans lesquels la notion d'environnement recevra avec J.C. Perrin une «épaisseur territoriale». 
Constitué en 1984, le GREMI a eu pour principal objectif «d'organiser et de coordonner des travaux menés par des équipes de recherche européennes de manière à mieux comprendre les processus, apprécier les pratiques et les politiques et, finalement, proposer des formes d'action, notamment locales, adaptées à l'objectif de diffusion de l'innovation technologique » (Aydalot, 1986). Un tel objectif exigeait préalablement une démarche appropriée. La particularité de celle-ci tient avant tout au choix méthodologique original qui révèle d'emblée la particularité du GREMI par rapport aux approches traditionnelles. Trois angles d'investigation de la relation espace-technologie pouvaient en effet être distingués :

1. Une entrée par les entreprises, orientée vers la détermination et l'examen des facteurs de localisation des activités haute technologie. Dans cette optique, l'espace est réduit à un « offreur » de facteurs de localisation.

2. Une entrée par la technologie. Il s'agit d'apprécier l'impact des technologies nouvelles sur le développement régional et l'évolution des disparités spatiales. En considérant la technologie comme exogène et donnée a priori, cette approche tend à privilégier l'analyse des résultats sur celle des processus d'innovation.

3. Une entrée par les milieux locaux, à travers laquelle sont appréciées leurs aptitudes à créer, développer et diffuser les innovations technologiques. Il s'agit d'étudier les conditions extérieures à l'entreprise favorisant la naissance et l'enracinement des firmes innovatrices.

C'est cette troisième approche que le GREMI a finalement décidé de privilégier. L'entreprise innovatrice «ne préexiste pas aux milieux locaux » : elle est "sécrétée » par eux. Aydalot (1986) pose l'hypothèse du rôle déterminant joué par les milieux locaux. Incubateurs de l'innovation et prisme à travers lequel passeront les incitations à l'innovation, ils donnent sur le terrain son visage à celle-ci. L'entreprise n'est pas un agent innovateur isolé : elle est partie du milieu qui la fait agir. Le passé des territoires, leur organisation, leurs comportements collectifs, le consensus qui les structure sont des composantes majeures de l'innovation. En d'autres termes, il s'agit de considérer les milieux industriels locaux comme essentiels dans la compréhension des formes et rythmes des processus d'innovation. Comme le note Aydalot, cela entraîne que « les comportements innovateurs ne sont pas nationaux, mais qu'ils dépendent de variables définies au niveau local ou régional ».

Nous pouvons brièvement résumer la problématique autour de quelques questions majeures. Quels sont les facteurs locaux d'innovation ? Pourquoi certains milieux innovent-ils plus que d'autres? Quel est le rôle de la proximité des acteurs et des synergies locales dans le processus d'innovation? 
Quelle est l'importance des facteurs extérieurs ? Les trajectoires d'apprentissage technologique des entreprises sont-elles liées à une organisation particulière du milieu local ? Dans quelle mesure la notion de milieu innovateur peut-elle contribuer à la reconstruction d'une théorie de la dynamique spatiale et ainsi définir de nouvelles orientations à la politique régionale ? Autant d'interrogations auxquelles le GREMI s'est fixé comme ambition d'apporter un certain nombre d'éléments de réponse en évitant soigneusement d'imposer un modèle unique et définitif.

La réflexion sera organisée autour de deux moments. Une première étape rappellera l'origine et le développement du concept de milieu innovateur à partir des différentes enquêtes de terrain menées par le GREMI. Cette mise en forme rétrospective des travaux empiriques permettra de souligner la particularité du concept de milieu innovateur dans l'approfondissement de la réflexion relative à la territorialisation des processus d'innovation technologique. Dans une seconde étape, l'unité théorique du concept de milieu et son apport dans la construction d'une problématique des dynamiques industrielles localisées seront examinés.

\section{Origine et développement du concept de milieu innovateur}

La reconnaissance des formes de territorialisation de la dynamique technologique a une histoire ancienne puisque la notion de pôle de croissance ou de développement, directement héritée des travaux précurseurs de François Perroux, reposait à la fois sur une approche en termes d'espace polarisé, avec diffusion hiérarchique, et sur l'impulsion technologique de la firme motrice. Plus récemment, dans le prolongement de la théorie de la polarisation des années 60 , les travaux à partir des notions de grappes technologiques, de technopoles, de spillovers interindustriels ont permis de s'interroger sur l'importance de la dimension spatiale dans l'émergence et le développement des processus d'innovation technologique. Cependant, comme l'ont montré Ravix et Torre (1991) ou Rallet (1991), à propos des technopoles, l'approche à partir de la notion de pôle de croissance, en dehors du fait qu'elle reste fortement attachée à une conception traditionnelle de la technologie, ne permet pas de penser la question de la territorialisation de la firme innovatrice en dehors d'une problématique de la juxtaposition spatiale des activités de R-D. C'est face à ces difficultés qu'il faut situer la démarche originale engagée depuis plusieurs années à partir du concept de milieu innovateur dans le cadre d'une problématique de l'organisation territoriale des processus d'innovation technologique. En distinguant les trois étapes de recherches menées par le GREMI, à la suite de l'organisation et de l'exploitation d'un travail minutieux 
d'investigation empirique commun à l'ensemble des équipes, sur la base d'une méthodologie unifiée et d'une grille de questionnement homogène, nous montrerons comment la prise en compte de la dimension territoriale interne à la dynamique technologique a progressivement permis de construire un concept de milieu innovateur qui endogénéise la technologie et le territoire dans une problématique de l'organisation et de la dynamique industrielle.

\subsection{L'aptitude des milieux industriels locaux à l'innovation : milieux innovateurs et trajectoires technologiques des entreprises}

La première étape correspond aux enquêtes GREMI I (Aydalot, 1986 ; Aydalot et Keeble, 1988) qui ont porté sur une quinzaine de régions européennes, comprenant des régions de vieille tradition industrielle (Newcastle, Charleroi, Saint-Étienne, Poznan), des régions de tradition industrielle dont le tissu productif est essentiellement composé de petites firmes (industrie horlogère et micro-technique de l'arc jurassien suisse et de Besançon), des régions métropolitaines (la cité scientifique Île-de-France Sud, Milan, Amsterdam), enfin des régions faiblement industrialisées (la zone Aix-Marseille, la région du Tessin en Suisse, Sophia-Antipolis). Le principal enseignement que l'on peut tirer de ces études de cas est le caractère distinctif des comportements et stratégies d'innovation. Ces travaux confirment, à des degrés divers, l'importance de l'environnement local et du contexte institutionnel dans les comportements d'innovation, faisant en définitive apparaître une différenciation spatiale des processus de création technologique. La diversité des milieux étudiés, liée en particulier à leur histoire industrielle, à leur niveau de développement, à leur structure sociale et politique, conduit à accepter une diversité des capacités à innover et des comportements innovateurs.

Cette diversité des formes localisées de dynamique technologique permet à Aydalot de construire une typologie des trajectoires technologiques. L'auteur distingue trois modes d'innovation technologique correspondant chacun à des logiques différentes d'acquisition des connaissances technologiques. Ces différents modèles conduisent à opposer trois formes spatiales de processus d'innovation ou types de milieu.

1. Une logique de reconversion d'un tissu industriel : la connaissance technologique est créée au sein du processus de production. Elle est donc interne à l'entreprise et propre au tissu industriel. Pour Aydalot, quelle que soit sa nature, l'innovation présente un caractère de « rupture-filiation » avec le tissu industriel existant. L'exemple retenu est celui de l'arc jurassien suisse. La tradition horlogère profondément 
ancrée dans le milieu industriel local a constitué le support d'une stratégie de diversification et de recomposition du système de production. Le glissement des qualifications mécaniques vers les qualifications électroniques, l'intégration progressive des produits électroniques issus de l'horlogerie à des usages nouveaux, l'association des technologies mécanique et électronique, la conception de produits nouveaux par l'utilisation et la valorisation de l'expérience et des savoir-faire issus de l'horlogerie illustrent le comportement innovateur par rupture-filiation d'un tissu de tradition industrielle dont la trajectoire technologique reste fortement influencée par les caractéristiques structurelles directement héritées du passé.

2. Une logique d'innovation par de grandes entreprises maîtrisant un champ nouveau : si la connaissance technologique reste interne à l'entreprise, elle est en revanche créée par des unités spécialisées disjointes du processus de production (laboratoires de recherche). Du point de vue de leur ancrage territorial, ces entreprises s'inscrivent dans une logique de division spatiale du travail guidée principalement par des facteurs d'attraction (infrastructure de transport et de communication, appareil de formation, qualité de la vie).

3. Enfin, une logique de création fondée sur la science : la connaissance technologique est élaborée hors de l'entreprise, dans des universités et des laboratoires de recherche publics. Cette connaissance est directement intégrée dans l'entreprise par des chercheurs créant leur propre entreprise ( «sous-produit » des centres de recherche) ou embauchés dans des entreprises existantes. Dans ce dernier modèle, la forme spatiale de l'innovation relève d'une logique de polarisation.

Ces premières enquêtes ont ainsi permis d'établir une relation entre la nature du processus d'innovation et la forme spatiale de l'innovation.

\subsection{Stratégies environnementales et différenciation des modes de territorialisation des firmes innovatrices}

Les enquêtes GREMI 2 (GREMI, 1988 ; Maillat et Perrin, 1992) marquent une nouvelle étape dans la connaissance des liens qui existent entre l'entreprise innovatrice et son environnement. Il s'agit d'étudier le rôle de l'environnement, plus précisément celui du milieu régional dans le développement du processus d'innovation au sein de l'entreprise. À cette fin, les enquêtes ont cherché à déterminer la nature des relations qu'une entreprise établit avec son environnement au cours de sa démarche d'innovation afin de mettre en 
évidence les modalités et les conditions d'enracinement des entreprises innovatrices dans leur milieu 5 .

Nous retiendrons de ces travaux trois principaux résultats.

1. La diversité des contextes territoriaux a permis de mettre en évidence la diversité des interactions entre les entreprises innovatrices et leur milieu, donc des formes de territorialisation des entreprises innovatrices. En particulier, ces enquêtes ont souligné l'importance des relations hors marché et des interdépendances, des formes localisées de coopération industrielle que les entreprises sont amenées à mettre en place dans leur démarche d'innovation.

2. Les modalités d'enracinement des entreprises innovatrices dans leur milieu local dépendent étroitement de la nature de leur stratégie d'innovation. Il convient à cet effet de reprendre la distinction fondamentale faite par Gaffard $(1986,1990)$ entre l'exploitation d'une trajectoire technologique, c'est-à-dire « un processus d'adaptation et de diffusion d'une technologie préexistante », et la création de technologie. Ces deux stratégies d'innovation correspondent à deux modes opposés de relation à l'environnement territorial. Dans le cas de l'exploitation d'une trajectoire technologique, les entreprises innovatrices accordent une faible importance aux liens locaux : l'environnement est traité comme une donnée exogène et la relation avec le milieu local répond « à la nature de la contrainte spatiale de disponibilité des ressources génériques »(Gaffard, 1986). En revanche, dans les stratégies de création de technologie, l'entreprise innovatrice se caractérise par une forte intégration au milieu local. L'enracinement territorial de la firme innovatrice participe au développement de savoir-faire collectifs spécifiques. Comme l'observe Gaffard, «la localisation est un élément important de la capacité d'échange d'information, et donc de la création de technologie ». Dès lors, « la localisation d'activités n'est plus subordonnée aux trajectoires technologiques, au contraire elle devient un élément de la stratégie

5. Les résultats, présentés au colloque GREMI (1988), ont été édités par D. Maillat et J.C. Perrin (1992). Les enquêtes ont porté sur une dizaine de régions d'Europe et des États-Unis, qui appartiennent à cinq contextes territoriaux différents : les régions métropolitaines (Ille-de-France, Vallès Oriental dans l'aire barcelonnaise, le secteur nord de la zone métropolitaine de Milan), les régions périmétropolitaines (Aix dans la périphérie de l'aire métropolitaine marseillaise, la région de Bergame dans l'aire milanaise), des régions de type « district industriel » (l'arc jurassien suisse), des régions de tradition industrielle (Liège), des régions en voie d'industrialisation (Tessin, Nice, Poitou-Charente), des régions technopolitaines (Silicon Valley, Sophia-Antipolis). 
de création et commande pour partie celle-ci » (1986). En d'autres termes, la proximité territoriale des différents partenaires engagés dans la démarche d'innovation est un élément fondamental dans le développement de coopérations créatrices de ressources particulières, notamment de resssources humaines. Dans ces stratégies de création de technologie, la territorialisation de la firme innovatrice se traduit par la construction d'un environnement particulier d'innovation. Cet environnement particulier, qui relève d'une démarche de nature procédurale, traduit cette dimension contextuelle de l'organisation des coopérations créatrices de savoir-faire d'innovation et des processus collectifs d'apprentissage (Perrin, 1991a).

3. Cette logique d'enracinement des entreprises innovatrices dans leur environnement local n'exclut en aucun cas les liaisons hors milieu. Nous retiendrons en effet des enquêtes l'importance des réseaux extra-territoriaux dans la démarche d'innovation. Celle-ci associe la logique de territorialisation à celle de l'ouverture. En définitive, la capacité d'innovation d'un milieu va simultanément dépendre de son degré de cohérence interne (les interdépendances de proximité) et de son degré d'ouverture (GREMI, 1989a).

\subsection{Milieux innovateurs et réseaux d'innovation}

En mettant l'accent sur les phénomènes de coopération et d'interdépendances, en soulignant l'importance de certaines formes particulières d'organisation territorialisées, la notion de réseau d'innovation, et par ce biais la dimension organisationnelle des processus de création de ressources, s'est imposée comme un champ d'investigation inséparable et complémentaire de la notion de milieu innovateur. Pour Perrin (1990a), « un réseau d'innovation est une forme d'organisation des relations entre les acteurs d'un processus d'innovation, qui, par sa durée et son ouverture (pluralité des spécialisations, diversité des savoir-faire), met en œuvre un apprentissage individuel et collectif dont l'effet synergétique contribue de manière déterminante à la créativité de l'ensemble ". À travers cette notion de réseau d'innovation, les enquêtes GREMI 3 (GREMI, 1990 ; Maillat, Quevit et Senn, 1993) se sont fixé comme principal objectif de mettre en évidence les aspects organisationnels des processus d'apprentissage collectif à la base d'une démarche d'innovation. Pour ce faire, ces enquêtes devaient répondre aux trois niveaux d'interrogations suivants :

- la genèse, le développement de formes organisationnelles spéciales appelées réseaux d'innovation ;

- le rôle du milieu dans l'émergence de ces formes organisationnelles ; 
- les interactions milieu-réseau : quel est le rôle du milieu dans l'organisation du réseau d'innovation ? Comment les réseaux d'innovation contribuent-ils à l'accroissement des capacités créatrices du milieu ?

Dans la lignée des travaux contemporains consacrés à l'analyse économique de la technologie, les travaux du GREMI ont confirmé certaines caractéristiques essentielles de la démarche d'innovation. Il s'agit d'un processus fondamentalement collectif qui procède de la combinaison créatrice de savoirfaire et de compétences spécifiques. Cette démarche créatrice relève d'un processus dynamique d'apprentissage, c'est-à-dire de spécification de ressources, et en particulier de ressources humaines. À ces caractéristiques principales, les recherches du GREMI ont permis de montrer que l'organisation territoriale est une composante essentielle de la création techno-économique. Compte tenu de ces éléments, on peut dès lors poser l'hypothèse que le marché ou la hiérarchie, dans le cadre de leur dynamique propre, se révèlent inadaptés pour donner vie au processus d'innovation. La démarche créatrice suppose qu'au préalable on ait implanté une innovation de type organisationnel : ce mode d'organisation particulier sera appelé réseau d'innovation ${ }^{6}$. Cette notion de réseau recouvre ici quatre dimensions principales :

- Une dimension économique : il s'agit d'un mode d'organisation hybride qui d'un point de vue théorique s'inscrit dans un dépassement de la dualité firme/marché.

- Une dimension historique : un réseau suppose un système de relations de long terme entre différents acteurs, basé sur des règles de confiance, de connaissances mutuelles, de réciprocité et de priorité. En d'autres termes, le réseau constitue un mode d'organisation des transactions qui se développe dans le temps et qui exprime l'existence de liens de fidélité et d'une préférence territoriale entre les différents partenaires (Lecoq, 1993).

- Une dimension cognitive : l'organisation réseau est dépositaire d'un savoir-faire collectif qui est supérieur à la somme des savoir-faire individuels. L'intérêt de ce mode d'organisation est de permettre le développement de processus d'apprentissage collectifs.

- Une dimension normative : le réseau se caractérise par un système propre de règles destiné à préciser les obligations et contraintes de

6. Sur cette question des réseaux d'innovation, on notera le numéro spécial de la revue Research Policy vol. 20, n 5, octobre 1991, qui reprend une partie des communications présentées au colloque international " Networks of Innovators " (Montréal, $1^{\text {cr }}$ et 2 mai 1990). Il est intéressant de noter la forte proximité analytique avec la problématique développée par le GREMI. 
chacun des partenaires. Ces règles permettent ainsi de délimiter un espace de travail collectif.

En définitive, le réseau d'innovation constitue une réponse organisationnelle appropriée à la complexité de la démarche d'innovation. Il détermine un espace de travail collectif approprié à la combinaison créatrice de savoir-faire et de compétences internes et externes à la firme. En précisant de la sorte la notion de réseau d'innovation, les enquêtes GREMI 3 ont centré leur attention sur la nature et les propriétés de ces relations de coopération entre firmes, en s'efforçant notamment de montrer combien la proximité spatiale des différents partenaires permet d'accroître leur capacité d'apprentissage.

Compte tenu des résultats obtenus, nous pouvons dégager deux enseignements majeurs :

- Les enquêtes ont tout d'abord mis en évidence une différenciation des formes de réseaux selon la stratégie technologique. Perrin (1990a) distingue ainsi deux catégories fondamentales de réseau d'innovation : des réseaux d'innovation par exploitation d'une trajectoire technologique (réseaux hiérarchiques) et des réseaux d'innovation par création de technologie (réseaux partenariaux). Dans le premier cas, il s'agit de réseaux structurés autour d'une firme leader qui contrôle l'ensemble de la démarche d'innovation, de la conception à l'industrialisation du produit ou procédé nouveau jusqu'à sa commercialisation. L'appel à des partenaires extérieurs se justifie principalement par la recherche de compétences complémentaires dont la maîtrise supposerait des investissements longs et coûteux. Les transactions entre le maître d'œuvre et les partenaires sont donc essentiellement bilatérales, définies dans le cadre d'un contrat. Dans cette organisation hiérarchisée, le milieu intervient principalement sur le plan de l'offre de ressources complémentaires génériques. Dans le cadre des réseaux de création de technologie ou réseaux partenariaux, la coordination centrale de la démarche d'innovation n'est pas assurée par une seule firme. En revanche, on observe une conception collective et globale du projet d'innovation. L'élément fédérateur des différents partenaires est la notion de projet : le réseau définit en priorité un espace de travail collectif et d'échange visant à construire une capacité continue et durable de création de ressources particulières et à maintenir une dynamique d'apprentissage.

Dans ce modèle, les transactions sont essentiellement multilatérales, réglées par des relations de confiance, de réciprocité, par une certaine éthique de travail liée à l'appartenance à un même milieu (Maillat, 
Crevoisier et Lecoq, 1991b)7 ${ }^{7}$. Il y a ainsi enracinement du réseau d'innovation dans un contexte territorial particulier. Dans ces conditions, le réseau d'innovation apparaît comme le contexte organisationnel d'une dynamique continue et durable d'apprentissage collectif des savoir-faire et de construction de ressources particulières; le milieu innovateur est le contexte approprié à la formation, au développement et la diffusion des réseaux d'innovation (Perrin, 1991a ; $1991 b)^{8}$.

- Enfin, ces travaux ont confirmé une étroite interdépendance entre, d'une part, la nature, les propriétés et le mode de fonctionnement du réseau d'innovation et, d'autre part, le milieu innovateur. La particularité des contextes locaux imprime des formes d'organisation en réseau spéciales qui vont engendrer des dynamiques d'apprentissage différenciées.

La contribution du GREMI à l'analyse des relations entre technologie et territoire nous amène à soumettre au moins trois observations critiques. Premièrement, l'approche par les milieux innovateurs semble aujourd'hui tiraillée entre, d'un côté, une perspective pointilliste des questions d'organisation industrielle localisée et la multiplicité de modèles locaux difficilement

7. Nous retrouvons chez B. Planque (1991) cette dualité dans l'opposition entre les réseaux d'innovation contractuels et les réseaux d'innovation conventionnels. Alors que les premiers sont des réseaux monofonctionnels, gérés par un contrat qui établit les objectifs (souvent limités), l'horizon temporel, les droits et sanctions des différents partenaires, les seconds sont des réseaux multifonctionnels de long terme qui se construisent sur une confiance mutuelle des acteurs. Pour B. Planque, ces réseaux d'innovation conventionnels «ne peuvent probablement exister que sous une forme territoriale polarisée qui assure la fréquence des relations, la connaissance réciproque continue des partenaires nécessaires au maintien et à l'adaptation temporelle commune de la convention ». Dans une perspective sensiblement convergente, D. Maillat et son équipe ont élargi la typologie en différenciant, sur la base de la nature des processus d'innovation et les modes de territorialisation de la firme innovatrice, les réseaux avec firme leader (réseaux hiérarchiques), les réseaux compacts (ou réseaux partenariaux) et les réseaux avec firme pivot que l'on peut considérer comme une forme hybride des deux types précédents (Maillat, Crevoisier et Lecoq, 1991b).

8. La notion de contexte ou d'organisation contextuelle (Perrin, 1991a) est directement empruntée aux travaux de K. Imai et Y. Baba $(1989,1990)$ sur la nature systémique et organisationnelle des processus d'innovation. Est qualifiée de « contextuelle » la forme d'organisation appropriée à une démarche de nature procédurale d'apprentissage collectif (learning by interacting). Dans une perspective très proche de celle du GREMI, on retrouve cette idée de contextualité chez B. Johannisson (1990a, 1990b) qui, pour étudier plus spécifiquement les conditions d'émergence d'un entrepreneurship, utilise les notions de «local context » ou encore de «socioeconomic spatial context». 
extrapolables et, de l'autre, l'enfermement d'une diversité de processus locaux dans un modèle unique du milieu innovateur. Deuxièmement, la problématique du milieu innovateur tend à systématiser et à surestimer le rôle de la proximité géographique et des synergies locales dans les processus d'innovation technologique. Rallet a largement montré dans un article récent (1993) la nécessité de relativiser ce rôle, réfutant l'idée « d'un déterminisme technologique de la proximité ». Troisièmement, enfin, la problématique des milieux innovateurs tend à négliger l'importance du temps long dans la construction des interactions spatiales. Non seulement elle ne prend pas suffisamment en compte l'histoire des firmes, des institutions, du milieu, dans l'analyse des processus de création de ressources nouvelles, mais elle tend également à négliger l'importance des prévisions, fondatrices des évolutions futures, dans la constitution des dynamiques industrielles localisées 9 .

\section{Des milieux innovateurs aux dynamiques industrielles localisées}

L'intégration de la dimension territoriale dans les stratégies technologiques des firmes à partir du concept de milieu innovateur représente une tentative féconde de croisement entre deux problématiques d'analyse du changement technique et d'économie régionale, restées jusqu'alors relativement indépendantes l'une de l'autre. L'enjeu théorique de cette démarche est de saisir la complexité des liens qui se développent entre dynamique industrielle et territoire à partir précisément du processus de construction territoriale de la firme innovatrice saisi par le concept de milieu innovateur. Après en avoir rappelé la nature et les fondements théoriques, nous nous proposons de dégager quelques perspectives de réflexion dans la construction d'une problématique des dynamiques industrielles localisées.

\subsection{Nature et fondements théoriques du milieu innovateur}

Les études empiriques ont permis une avancée significative dans la connaissance des processus et formes localisés d'innovation et de création de ressources technologiques particulières. Articulées autour des deux notions centrales de milieu innovateur et de réseaux d'innovation, ces recherches ont confirmé

9. La question des prévisions, optimistes ou pessimistes, a été introduite par Lechot, Lecoq et Pfister (1993) dans l'analyse comparative de deux milieux (l'industrie horlogère de l'arc jurassien suisse et français) dont les trajectoires d'évolution ont divergé à partir des années 60 . Cette question des prévisions et des représentations des agents locaux, quant à leur incidence sur la genèse et la pérennité des systèmes localisés de production et d'innovation, est également étudiée par A. Torre (1993) et C. Dupuy (1994). 
l'importance du contexte territorial et d'une certaine proximité entre les firmes dans le développement de relations de coopération durables et d'une dynamique collective d'apprentissage. Les milieux innovateurs apparaissent comme le contexte de l'apprentissage, de l'émergence et du développement de réseaux d'innovation. La notion de milieu innovateur appelle néanmoins quelques éclaircissements.

Jusqu'à présent, l'image analytique du milieu innovateur est principalement celle d'une « boîte noire », à l'intérieur de laquelle se développeraient un certain nombre de processus particuliers. Aussi, nous nous proposons, sur la base des différents travaux du GREMI, de préciser la nature de ce contexte territorial. À ce propos, nous retiendrons trois niveaux différents de lecture du milieu innovateur (Maillat, Crevoisier et Lecoq, 1991a).

\subsubsection{Une approche micro-analytique du milieu}

Elle est essentiellement centrée sur une problématique en termes d'incertitude, d'information et de coût de transaction. En suivant Camagni (1991), «le milieu est un opérateur collectif de réduction du degré statique et dynamique d'incertitude auquel sont confrontées les firmes par l'organisation tacite ou explicite d'interdépendances fonctionnelles et informationnelles des acteurs locaux, en assurant de manière informelle les fonctions de recherche, transmission, sélection, transcription, transformation et contrôle de l'information ». Dans cette perspective et dans un cadre spatial, le milieu peut être considéré comme une structure de gestion efficiente alternative au marché et à la hiérarchie en permettant notamment une réduction des coûts de transaction.

\subsubsection{Une approche cognitive du milieu}

Elle est principalement articulée autour des notions d'apprentissage, de savoirfaire et de culture industrielle. Selon Maillat, Crevoisier et al. (1991), « le milieu regroupe dans un tout cohérent un appareil productif, une culture technique et des acteurs. L'esprit d'entreprise, les pratiques organisationnelles, les comportements d'entreprises, la manière d'utiliser les techniques, d'appréhender le marché, le savoir-faire sont autant d'éléments qui sont à la fois parties intégrantes et parties constitutives du milieu. Le milieu se présente comme un processus de perception, de compréhension et d'actions continuelles ». Cette définition a le mérite de faire apparaître deux aspects essentiels d'un milieu : d'une part, elle présente le milieu comme un processus et non comme un ensemble statique de ressources génériques ; elle souligne, d'autre part, l'importance du marché local du travail. Ce dernier autorise la circulation et l'échange des savoir-faire, la transmission d'une culture technique historique- 
ment et localement accumulée, la mobilité des compétences et par conséquent la continuité et la reproduction du milieu lui-même. Les auteurs observent en effet qu'il existe une forte cohérence entre le savoir-faire, la culture technique et industrielle, la technologie, le capital productif, qui est le fait de l'efficacité allocative et dynamique du marché local du travail.

\subsubsection{Une approche organisationnelle du milieu}

Pour Quevit (1991), « le milieu est un mélange de formes d'organisation qui structurent les stratégies d'entreprises selon la double logique d'externalisation et d'intégration organique. La particularité du milieu innovateur est de générer des processus organisationnels qui s'articulent sur ces deux logiques pour permettre la rencontre de formes d'organisation territorialisées et de réseaux extra-territoriaux. Le concept de milieu se réfère à des systèmes d'acteurs et à des structures appréhendées dans leurs interactions réciproques. La composante organisationnelle qui structure ces échanges est une variable essentielle à la compréhension des mécanismes qui constituent le milieu. »

Dans le même ordre d'idées, Perrin (1991b) définit le milieu comme « un ensemble territorialisé dans lequel des réseaux innovateurs se développent par l'apprentissage que font leurs acteurs des transactions multilatérales génératrices d'externalités spécifiques à l'innovation et par la convergence des apprentissages vers des formes de plus en plus performantes de création technologique ». Le milieu innovateur apparaît comme le contexte organisationnel de création de ressources particulières.

Nous retiendrons de ces différents niveaux de lecture quatre composantes principales :

- Une dimension territoriale. Le milieu est un espace géographique sans frontière a priori, qui ne correspond pas à une région donnée au sens commun du terme, mais qui présente une forte unité et cohérence se traduisant en priorité par des comportements identifiables et particuliers.

- Une logique organisationnelle. Elle relève de la coopération : les acteurs d'un milieu coopèrent pour innover. Cette coopération s'inscrit dans la durée et nécessite une ouverture des différents acteurs les uns sur les autres. Cette logique d'organisation prend forme à travers un système complexe d'interdépendance et de réciprocité caractérisé par un tissu dense de réseaux et de relations hors marché.

- Une dynamique d'apprentissage. Elle traduit la capacité des acteurs à modifier leur comportement, à imaginer et créer de nouvelles combinaisons productives. Cette logique d'apprentissage porte aussi bien sur 
la formation de savoir-faire, la création de nouvelles formes techniques ou organisationnelles, la création de règles permettant de rechercher un équilibre entre coopération et concurrence, afin de construire un espace de travail collectif, que sur la connaissance des différents partenaires et des capacités du milieu.

- Une culture industrielle. Elle exprime la mémoire du milieu, l'héritage des pratiques passées et des savoir-faire aussi bien techniques qu'organisationnels. Cette culture industrielle recouvre un ensemble de pratiques professionnelles et de valeurs, une certaine éthique du travail, un code de conduite. Elle permet une certaine cohérence dans les comportements et assure la stabilité du milieu ${ }^{10}$. Il est essentiel de souligner ici que cette cohérence va bien au-delà du système de valeurs et des normes de comportements qui régulent les relations entre les acteurs. Cette cohérence se construit avant tout autour d'un « projet productif » qui mobilise l'ensemble des acteurs - firmes et institutions - dans une démarche collective de création de nouvelles ressources et options productives. C'est dire en définitive que la prospérité d'un système localisé de production tout aussi bien que son déclin, voire sa destruction, sont des phénomènes essentiellement collectifs. Perrin (1993) montre ainsi que dans plusieurs régions l'effondrement de la filière métallurgie - construction navale de la région de Newcastle, le déclin de la métallurgie - équipements lourds

10. Cette culture industrielle, professionnelle et technique a été particulièrement bien mise en évidence dans certains contextes territoriaux étudiés par les équipes du GREMI, notamment dans la Silicon Valley (Gordon, 1991) et l'arc jurassien suisse (Maillat, Crevoisier et Vasserot, 1991), même si ces auteurs préfèrent parler de système de représentation et de savoir-faire. Pour Perrin, cette culture est « une manifestation et un produit de la rationalité procédurale qu'une collectivité se forge » dans une démarche créatrice. Cette culture « contribue à la cohésion des groupes et à l'accroissement de leurs capacités. À travers leur culture, les milieux projettent, d'eux-mêmes, une image qui leur permet de s'identifier et, partant, d'accroître leur autonomie et donc la maîtrise de leur fonctionnement et de leur développement. Dans la pratique, celle-ci s'exerce au moyen de codes de conduite qui ne sont ni institutionnalisés, ni formellement définis et que l'on appelle des conventions » (Perrin, 1991a). Nous retrouvons enfin cette intuition chez Freeman dans le débat sur les réseaux d'innovateurs ou d'innovation, lequel observe notamment que, "cultural factors such as language, educational background, regional loyalties, shared ideologies and experiences and even common leisure interests continue to play an important role in networking. An appreciation of these sociological factors in both formal and informal networks is a necessary complement to narrower "economic" explanations and helps greatly to understand the importance of regional networks, geographical proximity and "national systems of innovation" "(Freeman, 1991). 
de la Wallonie ou, encore, le développement des industries microtechniques héritières des traditions et savoir-faire horlogers dans l'arc jurassien suisse - la capacité de changement structurel d'un système localisé de production relève moins du comportement isolé des firmes que de l'organisation et des stratégies imaginées et mises en œuvre collectivement par les acteurs, c'est-à-dire en fin de compte d'un processus de type milieu.

\subsection{Les dynamiques industrielles localisées : un champ de recherches en construction}

Par la richesse et la variété des études de cas, la fécondité conceptuelle et analytique dont le GREMI a été l'instigateur, les travaux dont nous venons de présenter les grandes lignes constituent une avancée importante dans la compréhension des formes localisées d'innovation. Sur la base d'une méthodologie commune à l'ensemble des équipes, l'originalité de la démarche GREMI est $\mathrm{d}$ 'avoir permis de développer une approche comparative des processus technologiques localisés. Sans conteste, les concepts de milieux innovateurs et de réseaux d'innovation ont conduit à éclairer le débat sur la relation entre dynamique d'innovation et développement territorial en soulignant notamment l'émergence de nouvelles formes d'organisation industrielle et l'importance de leur territorialisation (Gordon, 1990 ; Perrin, 1991a).

Plus fondamentalement, les recherches entreprises par le GREMI (1987, 1988, 1989a, 1989b, 1990, 1993) s'inscrivent dans une problématique plus large relative aux relations entre dynamique industrielle et dynamique des territoires ${ }^{11}$. C'est en effet le sens que donne Perrin (1990b) à l'ensemble de ces recherches empiriques comparatives. Il considère que l'intérêt majeur du concept de milieu innovateur « est de parvenir à une synthèse de l'approche industrielle et de l'approche spatiale-territoriale [...], d'associer étroitement la

11. L'enjeu théorique est clairement exposé par Perrin (1992) dans la synthèse qu'il propose des travaux GREMI 2 lorsqu'il écrit « que la dynamique socio-économique procède à la fois par création de technologies et par construction de " territoires " et que ces deux processus sont étroitement liés de sorte que, de même que la dynamique industrielle a un effet déterminant sur le développement des systèmes locaux-régionaux, ce qui est le plus souvent admis, de même, l'organisation territoriale des activités productives contribue de manière tout aussi importante à la progression de leurs capacités, notamment dans l'ordre technique-technologique, ce qui est rarement reconnu ». Dès lors, l'objectif du GREMI est de « s'efforcer de développer à partir d'études empiriques menées selon un même protocole d'enquête, dans un échantillon de régions suffisamment diversifié pour être significatif et par une démarche comparable systématique, une analyse intégrée de la dynamique industrielle et de la dynamique territoriale ». 
création technologique et la construction territoriale dans l'analyse de la dynamique industrielle et de montrer comment la prise en compte de la donnée spatiale-territoriale est susceptible d'enrichir celle-ci ». Il s'agit ainsi de restituer la composante territoriale de la création technologique et de la dynamique productive, renouant ainsi avec une longue tradition allant de l'analyse marshallienne des « districts manufacturiers » à Perroux qui, à travers son approche centrée sur le pôle de croissance et la firme motrice, avait sans doute posé les premiers éléments d'une analyse moderne de la composante territoriale de la dynamique industrielle, tradition qui trouve ses prolongements dans les interrogations récentes relatives à « la dimension territoriale de la coopération industrielle » (Gaffard, 1990) et dans les recherches consacrées aux systèmes localisés d'innovation (Gaffard et al., 1991). Dès lors, il n'est pas étonnant de relever une certaine continuité analytique entre les notions de district industriel, pôle de croissance, système localisé de production ou milieu innovateur, diversité de terminologie qui révèle la complexité des formes territoriales d'interdépendance hors marché et de coopération industrielle fondées sur la proximité spatiale des acteurs.

Avec les notions de milieu innovateur et de réseau d'innovation, les chercheurs du GREMI ont posé les premiers éléments d'une analyse économique de la proximité et de la coopération industrielle territoriale allant dans le sens de la construction d'une théorie des dynamiques industrielles localisées dans laquelle dynamique territoriale et dynamique technologique sont endogénéisées dans une problématique globale de création de ressources. En d'autres termes, à la différence d'une approche en termes de localisation centrée sur une problématique d'allocation de ressources génériques dans laquelle le territoire est réduit à un cadre récepteur dont les propriétés sont définies $a$ priori, le territoire résulte dorénavant d'un processus de construction issu des stratégies des acteurs et des phénomènes d'apprentissage collectif. Nous savons que ce programme de recherche n'en est qu'à ses débuts, et il reste beaucoup de chemin à parcourir pour disposer d'une formalisation satisfaisante des interdépendances entre les trois niveaux que sont la dynamique industrielle, la dynamique technologique et la dynamique territoriale. Si les approches axées sur le milieu innovateur ont permis une réelle avancée dans l'analyse économique des rapports entre territoire et technologie, nous pouvons cependant relever certaines insuffisances qui sont autant de directions de recherches à venir. À ce sujet, trois voies méritent certainement d'être explorées. Nous nous proposons d'en suggérer ici les grandes lignes directrices. Sur le plan des concepts tout d'abord, une clarification urgente s'impose. On peut en effet s'étonner de l'absence de cadre théorique unifié. Ainsi, il est tout à fait caractéristique de noter que les points d'appui théorique restent extérieurs au champ traditionnel de l'économie régionale, alors que ces travaux sont préci- 
sément, à l'origine, une initiative de chercheurs appartenant au courant de la science régionale. Observons également que la notion de milieu innovateur est loin d'apparaître comme isolée dans ce champ de recherches consacré aux dynamiques industrielles localisées. On relèvera l'extrême diversité de notions souvent concurrentes, allant du milieu innovateur au système d'innovation localisé, en passant par les districts industriels ou technologiques, sans oublier les premiers travaux consacrés au système productif régional. Peut-on opposer si facilement, comme il l'a souvent été fait, le district industriel au milieu innovateur sur la distinction entre une approche statique et une lecture dynamique $^{12}$ ? Sommes-nous certains de parler de la même chose ?

Une seconde piste de réflexion concerne la question des fondements micro-économiques de la rationalité des comportements des agents à l'intérieur des systèmes localisés de production et d'innovation. Les travaux sur les milieux innovateurs, rejoignant par là l'essentiel de la littérature italienne sur les districts industriels, n'ont pas manqué de souligner l'importance des phénomènes de confiance, de réciprocité, de solidarité dans les interdépendances hors marché qui se nouent localement entre les acteurs. Nous-même avions précédemment introduit les logiques de préférence territoriale et de liens de fidélité dans la rationalité de l'agent économique liées à son « ancrage » et à son appartenance à un même contexte territorial (Lecoq, 1993). En faisant de la confiance un mécanisme essentiel des modes de coordination dans les relations interfirmes, il nous semble important de réexaminer la question des mécanismes incitatifs et la nature des formes d'interdépendances entre les partenaires d'un même système localisé de production et d'innovation afin d'en dégager la rationalité économique.

Un dernier champ de recherches concerne l'évolution structurelle des milieux innovateurs, plus précisément la question de l'historicité des formes d'évolution des systèmes localisés de production et d'innovation. On peut s'étonner de l'absence d'une véritable approche de la dynamique historique et évolutive des milieux innovateurs. La question des trajectoires technologiques régionales ou de l'évolution de la territorialité industrielle, pour reprendre la

12. La différenciation entre le district industriel et le milieu innovateur se basant sur une opposition entre statique et dynamique ne nous semble pas seulement stérile, mais surtout sans réel fondement. À notre sens, les analyses en termes de milieux innovateurs et de districts industriels illustrent plus fondamentalement deux modes distincts d'approche d'une même réalité, difficilement saisissable avec les découpages traditionnels de l'analyse économique, celle des systèmes localisés de production et d'innovation, étudiés sous deux angles différents et selon une méthodologie propre à chacune des approches. La définition que donne récemment G. Becattini (1992) du district industriel comme milieu créatif nous semble donc particulièrement caractéristique de la nécessaire complémentarité des différents modes d'investigation. 
terminologie employée par Perrin (1991a), demeure largement ignorée en dépit de quelques investigations dans cette direction (GREMI, 1989b; GREMI, 1993). L'introduction de la dimension historique dans l'analyse des milieux innovateurs ne vise pas seulement à enrichir la réflexion dans la reconnaissance d'une pluralité de mode de coordination et d'interdépendances localisées et l'examen des stratégies différenciées de territorialisation des firmes innovatrices. Cette dimension historique invite principalement au glissement d'une problématique dont l'objet scientifique ne se réduit plus à un ensemble d'interrogations relatives au mode de fonctionnement des systèmes localisés d'innovation, mais plus fondamentalement introduit la question de l'origine des changements qualitatifs, de l'émergence et de la formation des milieux innovateurs. Il s'agit in fine de s'interroger sur la pérennité de ces systèmes localisés de production et d'innovation, de comprendre comment un milieu devient innovateur, renouvelle sa capacité de création de ressources particulières. L'investigation historique doit nous permettre de procéder à une analyse économique des milieux innovateurs dans la longue période. Il ne s'agit pas de dater notre discours en recherchant un temps chronologique, mais de restituer la variabilité historique de notre objet d'analyse, c'est-à-dire de lui rendre « l'épaisseur de la longue durée, l'intégrer dans le mouvement social, le jeu des conflictualités et dans une temporalité de l'irréversibilité, celle de l'apprentissage et de la mémoire » (Dockes et Rosier, 1991). Cette variabilité historique nous permet ainsi de justifier une diversité de formes localisées de systèmes d'innovation, diversité qui renvoie à des processus historiques, hors de l'équilibre, de construction de ces espaces. La question de l'évolution des milieux innovateurs s'inscrit dès lors dans une analyse économique du changement dans un temps historique, analyse dans laquelle le territoire ne peut être réduit à un ensemble de facteurs de localisation donnés a priori, mais est entendu comme ressource spécifique dont la construction est un élément essentiel d'un processus global de changement (Gaffard, 1992) ${ }^{13}$.

Ces différentes perspectives d'investigation révèlent de toute évidence qu'il existe là un champ de recherches encore largement en friche mais qui ne saurait être négligé dans la construction d'une analyse des dynamiques industrielles localisées. En se situant dans la perspective d'une analyse économique de la longue période, les dernières contributions du Groupe de recherche sur

13. Cette épaisseur historique des processus est clairement affirmée par G. Becattini (1990) lorsqu'il définit notamment le district industriel comme « une entité socioterritoriale caractérisée par la présence active d'une communauté de personnes et d'une population d'entreprises dans un espace géographique et historique donné [...]. Le terme localisation va bien au-delà de la concentration accidentelle dans un même lieu d'un processus de production attiré par des facteurs de localisation préexistants. Les entreprises s'enracinent au contraire dans le territoire et ce processus ne peut être conceptualisé indépendamment de son évolution historique. » 
les milieux innovateurs (GREMI, 1993) ont permis de poser les premiers éléments d'une analyse réellement dynamique, dans laquelle l'étude des processus d'innovation sur le plan local acquiert une véritable épaisseur temporelle. Ainsi, l'accent est porté analytiquement non plus sur un point d'arrivée - le milieu innovateur considéré comme une boîte noire constituée et stable-, mais sur la capacité d'un système local à construire chemin faisant de nouvelles options productives et à s'engager dans un processus global de changement défini comme un processus de destruction créatrice au sens de J. Schumpeter ou de rupture-filiation dans les termes de Aydalot.

\section{Conclusion}

La construction du concept de milieu innovateur pour qualifier les articulations entre les logiques industrielles, technologiques et spatiales a permis une réelle avancée dans la connaissance des formes d'organisation spatiales de la dynamique technologique. Les réflexions engagées depuis une dizaine d'années, à la fois sur les plans empirique et théorique, ont permis de dépasser cette représentation traditionnelle de l'espace comme simple cadre de diffusion des technologies pour ouvrir sur une problématique de la localisation de la firme entendue comme vecteur essentiel de l'émergence et du développement des processus d'innovation. L'approche par les milieux innovateurs a ainsi conduit à souligner l'importance des formes spatiales d'interdépendance fondées sur la proximité des acteurs dans la mise en œuvre de processus d'innovation technologique, c'est-à-dire de processus collectifs de recherche et d'apprentissage de nouvelles solutions productives. Le processus de localisation de la firme innovatrice - entendu ici comme processus de construction territoriale - devient un élément essentiel dans la mise en œuvre et la viabilité d'une démarche de création de nouvelles options productives.

Au-delà de la diversité des formes spatiales de la dynamique technologique, l'approche par les milieux innovateurs permet d'avancer quelques propositions novatrices que l'on peut brièvement structurer autour de quatre idées directrices.

Premièrement, le concept de milieu innovateur permet de comprendre la localisation des firmes non plus comme un état mais comme un processus. $\mathrm{La}$ localisation de la firme innovatrice est un processus essentiellement dynamique. Elle ne se réduit donc pas à un moment particulier de la vie de l'entreprise - l'adoption d'un site d'implantation - mais se construit dans le temps. Le problème économique se déplace de la rationalité d'un choix entre des alternatives données (la décision d'adoption d'un site particulier entre différentes alternatives) vers la viabilité d'un processus qui s'inscrit dans une démarche procédurale de création de nouvelles options productives. 
Deuxièmement, la firme innovatrice construit sa localisation en même temps qu'elle imagine et élabore de nouvelles solutions et opportunités productives. La localisation de la firme innovatrice devient donc inséparable analytiquement du processus de production entendu dans son acceptation la plus large, c'est-à-dire verticalement intégré et considéré comme un tout dans le temps.

Troisièmement, le processus de localisation est analysé comme un processus d'apprentissage. Autrement dit, l'analyse d'un système localisé de production et d'innovation prend place dans une analyse économique d'un processus de changement dont la dimension principale est celle du temps. La localisation de la firme innovatrice est un processus de nature séquentielle, qui se construit au fur et à mesure des contraintes et des opportunités rencontrées par les acteurs.

Quatrièmement, enfin, l'approche par les milieux innovateurs suggère que le territoire économique n'est pas donné a priori. Il est construit par les firmes dans le cadre d'une dynamique de création de ressources spécifiques. Nous rejoignons sur ce plan le point de vue défendu par Rallet (1993) pour lequel « l'existence du territoire n'est pas structurellement garantie. Il est le résultat d'une construction, l'effet d'engagements territoriaux des agents et des institutions et peut, à ce titre, tout aussi bien se défaire que se faire ». Cette dernière proposition nous permet de considérer l'organisation territoriale des acteurs comme une composante active de la dynamique économique et notamment des processus de production et d'innovation. Elle suggère enfin que la dynamique spatiale est donnée par l'articulation intertemporelle des séquences de construction territoriale et destruction territoriale engagées par les firmes innovatrices dans le cadre des processus de création de nouvelles ressources. 


\section{Bibliographie}

Aydalot, P. (dir.) (1986), Milieux innovateurs en Europe, Paris, GREMI.

Aydalot, P. et D. KEeBle, (dir.) (1988), High Technology Industry and Innovative Environment: The European Experience, Londres, Routledge.

BECATtini, G. (1990), «The Marshallian industrial district as a socio-economic notion », dans F. Pyke, G. Becattini et W. Sengenberger, Industrial Districts and Inter-firm Co-operation in Italy, London, International Institute for Labour Studies, p. 37-51.

BeCATtini, G. (1992), «Le district industriel : milieu créatif », Espaces et Sociétés, $\mathrm{n}^{\circ}$ 66-67, p. 147-163.

BELLET, M. (1992), « Technologie et territoire : l'organisation comme objet de recherche ? ", Revue Française d'Économie, vol. 7, n 1, p. 85-138.

Bellet, M., G. Colletis, B. Lecoq, Y. Lung, B. Pecqueur, A. Rallet et A. TORRE (1992), «Et pourtant ça marche! Quelques réflexions sur l'analyse du concept de proximité », Revue d'Economie Industrielle, vol. 61, $\mathrm{n}^{\circ} 3$, p. $111-128$.

CAMAgni, R. (1991), Innovation Networks - Spatial Perspective, Londres, Belhaven Press.

CAMAGNI, R. (1991), « Local milieu, uncertainty and innovation networks : towards a new dynamic theory of economic space», dans Camagni, op. cit., p. $121-144$.

CRevoisier, O. et D. Maillat (1991), « Milieu, industrial organization and territorial production system : towards a new theory of spatial development », dans Camagni, p. 13-34.

Dockes, P. et B. Rosier (1991), « Histoire “ raisonnée” et économie historique », Revue Économique, vol. 42, $\mathrm{n}^{\circ} 2$, p. 181-208.

DUPUY, C. (1994), «Anticipations de comportements, conventions et dynamiques collectives de proximité », Revue d'Économie Régionale et Urbaine, à paraître.

FREEMAN, C. (1991), « Networks of innovators : a synthesis of research issues », Research Policy, vol. 20, $\mathrm{n}^{\circ}$ 5, p. 499-514.

GAFFARD, J.L. (1986), «Restructuration de l'espace économique et trajectoires technologiques », dans Aydalot, op. cit., p. 17-27.

GAFFARD, J.L. (1990), Économie industrielle et de l'innovation, Paris, Dalloz.

Gaffard, J.L., C. Charbit, J.C. Perrin, M. Quere et J.L. Ravix (1991), Cohérence et diversité des systèmes d'innovation : le cas des systèmes d'innovation localisés en Europe, Rapport réalisé dans le cadre du projet MUST, programme FAST (CEE), CNRS-LATAPSES, Université de Nice, février. 
GAFFARD, J.L. (1992), «Territory as a specific resource : the process of construction of local systems of innovation ", Communication au Séminaire de l'Università degli Studi di Siena, Siena, mars.

GREMI (1987), « Les politiques d'innovation technologique au niveau local. Articulation des dynamiques locales aux dynamiques externes », Actes de la TableRonde du GREMI, Paris, 14 et 15 décembre.

GREMI (1988), «Innovation et territorialisation des entreprises », Actes du colloque GREMI, Ascona (Suisse), 14 au 17 avril.

GREMI (1989a), «Milieux innovateurs et réseaux transnationaux : vers une nouvelle théorie du développement spatial », Actes du Colloque GREMI-EADA, Barcelone, 28 et 29 mars.

GREMI (1989b), « Trajectoires régionales de développement et achèvement du marché intérieur européen », Actes du Colloque Européen du GREMI, GREMIRIDER, Louvain-la-Neuve, 20 et 21 octobre.

GREMI (1990), « Nouvelles formes d'organisation industrielle : réseaux d'innovation et milieux locaux », Actes du Colloque GREMI, Neuchâtel (Suisse), 11 et 12 novembre.

GREMI (1993), « Dynamique d'ajustement structurel des milieux », Actes du Colloque GREMI, Ascona (Suisse), 29 au 31 octobre.

GORDON, R. (1990), "Systèmes de production, réseaux industriels et régions : les transformations dans l'organisation sociale et spatiale de l'innovation ", Revue d'Économie Industrielle, vol. 51, p. 304-339.

Gordon, R. (1991), « PME, réseau d'innovation et milieu technopolitain : la Silicon Valley », dans D. Maillat et J.C. Perrin, p. 195-220.

IMAI, K. et Y. BABA (1989), «Systemic innovation and cross-border networks. Transcending markets and hierarchies to create a new techno-economic system », Communication au International Seminar on the Contributions of Science and Technology to Economic Growth, OCDE, Paris, juin.

IMAI, K. et Y. BABA (1990), Systemic Innovation and Cross-Border Networks : The Case of the Evolution of the VCR Systems, Miméo, National Institute of Science and Technology Policy, Japon.

JOHANNISSON, B. (1990a), « Building an entrepreneurial career in a mixed economy : need for social and business ties in personal networks ", Communication à l'Academy of Management Annual Meeting, San Francisco, 12 au 15 août.

JOHANNISSON, B. (1990b), «Community entrepreneurship - cases and conceptualization », Entrepreneurship and Regional Development, vol. 2, p. 71-88. 
Lechot, G., B. LeCoQ et M. Pfister (1993), « Analyse comparative de l'évolution structurelle des milieux : le cas de l'industrie horlogère dans l'arc jurassien suisse et français », présenté au colloque GREMI IV, Dynamique d'ajustement structurel des milieux, Ascona (Suisse), 21 au 31 octobre.

LECOQ, B. (1993), «Proximité et rationalité économique », Revue d'Économie Régionale et Urbaine, $\mathrm{n}^{\circ} 3$, p. 469-486.

Maillat, D., O. Crevoisier et B. LeCoQ (1991a), Introduction à une approche quantitative du milieu, IRER. Neuchâtel (Suisse) : Working Paper 9102.

Maillat, D., O. Crevoisier et B. Lecoq (1991b), «Réseaux d'innovation et dynamique territoriale. Un essai de typologie », Revue d'Économie Régionale et Urbaine, $\mathrm{n}^{\text {os }} 3-4$, p. 407-432.

Maillat, D., O. Crevoisier et J.Y. VASSERot (1991), « Innovation et district industriel : l'arc jurassien suisse », dans Maillat et Perrin, op. cit., p. 105-124.

Maillat, D. et B. LeCOQ (1992), « New technologies and the transformation of regional structures in Europe : the role of the milieu », Entrepreneurship and Regional Development, vol. 4, p. 1-20.

MAILlat, D. et J.C. PERRIN (dir.) (1992), Entreprises innovatrices et développement territorial, Neuchâtel, EDES.

Maillat, D., M. QUevit et L. SENN (dir.) (1993), Réseaux d'innovation et milieux innovateurs : un pari pour le développement régional, Neufchâtel, EDES.

PERRIN, J.C. (1990a), « Réseaux d'innovation : contribution à une typologie », Communication au Colloque CREDIT-HEC, Networks of Innovators, Montréal, $1^{\mathrm{cr}}$ au 3 mai 1990, publiée dans les Notes de recherches du Centre d'Économie Régionale, 115, Aix-en-Provence.

PERRIN, J.C. (1990b), « Organisation industrielle : la composante territoriale », Revue d'Économie Industrielle, $\mathrm{n}^{\circ} 51$, p. 276-303.

PERRIN, J.C. (1991a), « Réseaux d'innovation, milieux innovateurs et développement territorial », Revue d'Économie Régionale et Urbaine, $\mathrm{n}^{\mathrm{os}}$ 3-4, p. 343-374.

PERRIN, J.C. (1991b), «Technological innovation and territorial development : an approach in terms of networks and milieu », dans Camagni, op. cit., p. 35-54.

PERrin, J.C. (1992), « Dynamique industrielle et développement local : un bilan en termes de milieux », dans Maillat et Perrin, op. cit., p. 223-255.

PERRIN, J.C. (1993), « Décentralisation et milieux locaux : étude comparative », dans Industrie et territoires en France, Paris, Les Études de la Documentation française, p. 71-93. 
Planque, B. (1991), « Note sur la notion de réseaux d'innovation. Réseaux contractuels et réseaux conventionnels ", Revue d'Économie Régionale et Urbaine, $\mathrm{n}^{\mathrm{os}} 3-4$, p. 295-320.

QUEvIT, M. (1991), «Innovative environments and local/international linkages in enterprise strategy : a framework for analysis », dans Camagni, op. cit., p. 55-70.

Rallet, A. (1991), «Théorie de la polarisation et technopoles », Économies et Sociétés, série «Progrès et Croissance », F, n 32, p. 43-64.

RALlet, A. (1993), « Choix de proximité et processus d'innovation technologique », Revue d'Économie Régionale et Urbaine, $\mathrm{n}^{\circ} 3$, p. 365-386.

RAVIX, J.L. et A. TORRE (1991), «Éléments pour une analyse industrielle des systèmes localisés de production ", Revue d'Économie Régionale et Urbaine, $\mathrm{n}^{\text {os }} 3-4$, p. $375-390$.

STÖRH, W.B. (1986), « Territorial innovation complexes », dans P. Aydalot, p. 29-54.

TORRE, A. (1993), "Proximité géographique et dynamiques industrielles », Revue d'Économie Régionale et Urbaine, $\mathrm{n}^{\circ} 3$, p. 432-448. 